\title{
Fortbildung verändert sich?!
}

_ Ist die Ausbildung als Physiotherapeut abgeschlossen, ist das Lernen noch nicht vorbei. Für manche mag das wie eine Binsenweisheit klingen. Denn gerade in der Physiotherapie ist Fortbildung ein zentrales Thema. Aktuell kommt die Fortbildungslandschaft in Bewegung. Traditionelle Therapiekonzepte arbeiten an ihren Inhalten. So erklärt die Bobath-Instruktorin Gabriele Eckhardt auf die Frage, ob sich die Kursteilnehmer auf neue Inhalte einstellen müssen, kurz und knapp: Ja! (॰S. 10).

_ Doch nicht nur mit Inhalten kann man Fortbildungen verändern - auch organisatorisch: Wie das gehen könnte, zeigt ein Blick über den großen Teich. In einer kürzlich erschienenen Studie erklärt der US-amerikanische Physiotherapeut Joshua Cleland, wie eine Fortbildung für Physiotherapeuten organisiert sein kann, um am Ende vor allem einen höheren Therapieerfolg für die Patienten zu erzielen. Für Cleland ist eine Fortbildung also dann effektiv, wenn die Patienten besser behandelt werden. Sein Ergebnis: Besuchen Physiotherapeuten eine Fortbildung, die ein anschließendes Training, z. B. in einer Übungsgruppe in der Klinik, beinhaltet, sind die Therapieerfolge bei den Patienten höher als bei Therapeuten, die „nur“ eine klassische Fortbildung ohne weiteres Training besucht haben. Ich bin gespannt, ob sich solche Ergebnisse der Bildungsforschung auf Fortbildungen auswirken werden.

_ Bildungsforschung klingt zwar wenig attraktiv. Doch gerade hier liegt die Chance. Denn Professionalität bedeutet für einen Berufsstand nicht nur, seine Therapie wissenschaftlich zu untermauern, sondern auch die durch Forschung erzielten Erkenntnisse effektiv im Unterricht zu vermitteln - mit dem Ziel, die Patientenversorgung zu verbessern. Es reicht also noch nicht, wenn wir wissen, was wirkt. Erst wenn dies in Aus- und Weiterbildung sinnvoll vermittelt wird, kann sich das positiv auf unsere Patienten auswirken.

_ Ich wünsche Ihnen viele positive Erfahrungen mit Fortbildungen, und genießen Sie die Sommerzeit!

Herzlichst, Ihr

\section{PHYSIOBONUS}

physiobonus heißen die

Gewinnspiele in physiopraxis.

Wer teilnehmen möchte, nutzt das Formular unter www.thieme.de/ physioonline in der Rubrik physioexklusiv. Und das gibt es in dieser Ausgabe zu gewinnen:

Kursplatz

1 Kurs „Triggerpunktbehandlung - Schulter"

Seite 71

Bücher

3-mal „Physiotherapie in der Gynäkologie“

3-mal „Die homöopathische Hausapotheke“

Seite 32

20-mal

„Körper, Seele, Mensch“

Seite 39

5-mal „Physiolexikon“

Seite 89

und außerdem

1 Paket mit 4 Rollen „K-Tape“ und einer Tapingschere Seite 44

2 Therapiekreisel Seite 50

1 sechsteiliger Muskelarm Seite 89

1 Balancierbrett

Seite 89
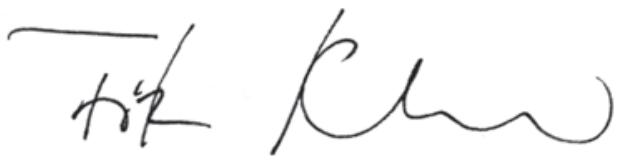